\title{
Wie Matrix und biologisches Rhythmussystem miteinander verknüpft sind
}

Schlaflosigkeit, Winterdepressionen, verminderte Leistungsfähigkeit und Verhaltensauffälligkeiten. Das sind nur einige Beispiele für Störungen, die entstehen, wenn die Rhythmen im menschlichen Körper aus dem Takt geraten. Denn gesund und leistungsfähig ist unser Organismus nur dann, wenn all seine Funktionen synchron aufeinander abgestimmt sind. Doch welche Funktionen sind im Körper eigentlich wie getaktet? Welche Rolle spielt dabei die Matrix? Und ist Stress immer ungesund?

Die Medizin hat erkannt, dass der Einfluss von Stress bei der Entstehung chronischer Erkrankungen eine entscheidende Rolle spielt. Man kann aber auch einen Schritt weiter gehen und sagen: Stress ist der Weg in die Gesundheit wie in die Krankheit. Doch warum ist das so?

\section{Regenerationsphasen sind das A \& 0}

Jeder Organismus zeigt seine Lebendigkeit in der Variabilität und Adaptionsfähigkeit seiner Rhythmen und Funktionen auf Reizeinwirkung (Stressoren). Die einzelnen systemspezifischen biologischen Rhythmen verändern sich dabei zueinander. Beobachtet man das Phänomen Stress einmal genauer, findet man immer einen endogenen Stressor (z. B. Störfeld, Ängste, Hypertonie) und/oder exogenen Stressor (z. B. Kälte, Lärm, Arbeitsüberlastung) als Auslöser komplexer Stressprozesse im Organismus. Diese Stressprozesse setzt der Organismus gezielt als Reaktion auf den Stressor ein, um eine Antwort für ihn zu finden und ihm diese mitzuteilen. Diesen evolutionären Prozessablauf beschrieb Virchow 1869 als einen Akkommodationsprozess: Bei funktionierendem Verlauf führt er immer zum Wohlfühlen, verstan- den als Gesundheit; bei nicht ausreichendem Verlauf dagegen immer zum Unwohlsein, verstanden als Krankheit.

Überfordern übermäßig intensive und lang anhaltende Reizeinwirkungen (z. B. hohes Arbeitspensum, psychische oder physische Dauerbelastung, Störfeldbelastung) die Adaptationsfähigkeit des Organismus, entsteht Stress: Die Matrix verliert zunehmend ihre Fähigkeit, in den Zustand der Ruhe-Rhythmik zurückzufinden - und damit die Fähigkeit der Regeneration.

Dauerstress von gleichbleibender Intensität oder starke kurzfristige psycho-physische Traumata stören das Zusammenspiel einzelner Rhythmen anhaltend und führen letztlich zum Verlust der Selbstordnungsfähigkeit und Anpassungsfähigkeit innerhalb des Grundregulationssystems: Es kommt zur Regulationsstarre oder Therapieresistenz. Vergleicht man das harmonisch aufeinander abgestimmte, kohärente Zusammenspiel aller biologischer Rhythmen mit einem Orchester, so klingt das Orchester Mensch unter Stress disharmonisch.

Aus dieser Sichtweise betrachtet regeln alle Kommunikations-, Informations- und Regelungsprobleme innerhalb der Matrix den labilen Gleichgewichtszustand zwischen Gesundheit und Krankheit. Somit bildet das Bindegewebe den zentralen Schauplatz lebenswichtiger Prozesse - gesteuert und erhalten durch einen gesunden und tragfähigen Rhythmus, der unter Stress sowohl adaptiv als auch destruktiv reagieren kann (A. Heinen). Therapeutisch ist es somit unerlässlich, Diagnose- und Therapieverfahren zu etablieren, die Aussagen zu den Rhythmen im Körper geben können bzw. sie beeinflussen.

\section{Nix geht ohne Matrix}

Die Summe der Bestandteile des Grundsystems steuert im Rhythmus die Homöodynamik. Sie bildet also das übergeordnete Ordnungsprinzip im Bestreben des Kör-

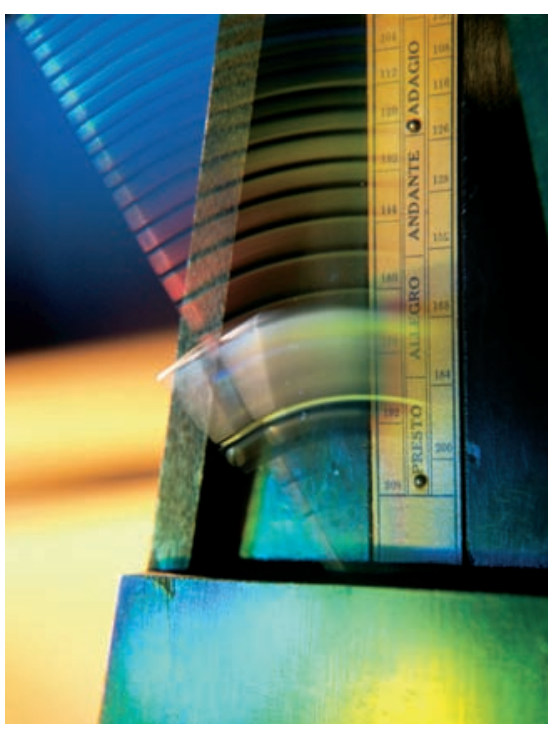

Abb. 1 Unser Biologischer-Ruhe-AktivitätsCyklus (BRAC) folgt einem festen zeitlichen Ablauf. Störungen können krank machen. Foto: (c) PhotoDisc

pers, sich selbst zu erhalten bzw. sich anzupassen. Alle biologischen Grundfunktionen des Lebens, die mit der Abwehr oder dem Ausgleich von Ungleichgewichten zusammenhängen, werden hier reguliert: Jeder Reiz und jeder Austausch zwischen den Organzellen verläuft über dieses Grundsystem, jede Reaktion des Nerven-, Gefäß-, Hormon- oder Immunsystems hängt von der Reaktionsfähigkeit dieser Matrix ab. Somit ist jegliche Antwort des Organismus auf einen physiologischen oder pathologischen Reiz an die Funktion des Grundsystems gekoppelt.

Diese holistische Sichtweise steht konträr zu dem über hundertjährigem Bestreben der Medizin, den Körper als ein beliebig teilbares Objekt zu betrachten und die Kausa der Krankheiten in singulären einzelnen Zellkompartimenten zu suchen und zu begründen. Was die Matrix in diesem Zusammenhang leistet, ist die Synthese einzelner Funktionen zu einem operierenden Ganzen. 
Es ist daher sinnvoll, diese holistische Betrachtungsweise in der Beschreibung der Matrix weiter zu verfolgen und die folgenden Bereiche im Hinblick auf die Rhythmen und den Einfluss von Stress einmal näher zu betrachten:

- Kohärenz und Integration
- Spannungsfeld der Matrix
- Inhärenter Zellstoffwechsel

\section{Kohärenz und Integration}

Netzwerkstrukturen sind nicht nur in der Geschäftswelt sinnvoll, sondern gerade für den zellulären Mikrokosmos bedeutsam. Die Vernetzung der Zellen untereinander erlaubt den Austausch aller nötigen Informationen an jedem beliebigen Ort und zu jeder beliebigen Zeit. Eine hohe Kohärenz liegt vor, wenn alle Zellen sich auf gleichem Informationsstand befinden. Daher sind lebendige Systeme Zustände der Kohärenz.

Der Zweck der Kohärenz liegt in der Vereinfachung. Sie entsteht nur dann, wenn alle überflüssigen Informationen, die das System belasten, wieder aufgelöst werden. Im Grundregulationssystem existiert ein ständiger Rhythmus der Neubildung und Wiederauflösung von Strukturen mit einer hohen Dynamik und dem klaren Ziel, alles Überflüssige und Schadhafte zu eliminieren. Leere Speicher z. B. werden umgehend aufgefüllt und neuen Anforderungen entsprechend mit einem angepassten Inhalt wieder bereitgestellt. Abfallstoffe werden umgehend bis auf die kleinste Ebene abgebaut und mit den Schadstoffen zur Ausscheidung gebracht. Unter therapeutischen Gesichtspunkten verliert eine „vergiftete“ Matrix deutlich an Kohärenz. Ist nun die Vergiftung das Übel oder die fehlende Kohärenz?

\section{Spannungsfeld der Matrix - Erklärungsmodelle für die Informa- tionstherapien}

Die „Ursuppe“ Matrix hat nicht nur einen „nährenden“ Charakter. Sie zeigt auch deutliche elektromagnetische Spannungsunterschiede auf. Das interzelluläre Bindegewebe funktioniert in seiner Gesamtheit als ein Di-Elektrikum: Es hält hohe Spannungen verlustfrei aufrecht. Dadurch können Reize, z. B. von Therapeutika eingebracht, erst erfolgreich zu Regenerationsprozessen verarbeitet werden bzw. zu Heilung führen. Diese elektrophysiologischen Zusammenhänge innerhalb der Matrix re- geln somit den Elektronenfluss innerhalb des Organismus. Alle mechanischen Reize lösen beispielsweise piezoelektrische Effekte (Änderung der elektrischen Polarisation und somit Änderung des Auftretens einer elektrischen Spannung an Festkörpern, wenn sie elastisch verformt werden) im Gewebe aus, die dann einen gerichteten Elektronenfluss zur Folge haben. Das Wirkungsspektrum dieser freien Elektronen reicht von einer Aktivierung des anabolen Stoffwechsels über die Neutralisation freier Radikale bis hin zur Regulation der Säurevalenzen im Gewebe - im Gegensatz zu den positiv geladenen Protonen, die durch die Bildung von Potenzialfeldern strukturgebend und richtungsweisend für die Zellteilungsprozesse sind.

In der Forschung werden die Elektronen weiterhin als universelle Energie- und Informationsträger angesehen und spielen für die Erklärung von Wirksamkeiten in der Informationsmedizin mittlerweile eine große Rolle (z. B. Homöopathie, Bioresonanz, Akupunktur etc.). Ebenso bestimmen die Anwesenheit und das Verhältnis der Elektrolyte Kalium/Natrium und Magnesium/ Kalzium maßgeblich den Sol- oder Gelzustand des Grundregulationssystems. Somit entscheidet das Verhältnis der elektrisch geladenen Teilchen, das Spannungsfeld der Matrix, über das Säuren-Basen-Gleichgewicht und die oxidative und/oder reduktive Ausrichtung der Stoffwechselprozesse.

\section{Inhärenter Zellstoffwechsel}

Wissenschaftliche Arbeiten von Jürgen Schole beschreiben weitere integrative $\mathrm{Zu}$ sammenhänge für das Bindegewebssystem. So unterliegen alle Körperzellen extra- wie intrazellulär den gleichen Gesetzen der Stoffwechselregulation. Unter Stoffwechsel versteht man den Transport und die chemische Umwandlung von Stoffen in einem Organismus. In diesen Stoffwechselvorgängen werden Wasser, Kohlenhydrate, Proteine, Fette und andere Ausgangsstoffe in chemische Verbindungen umgewandelt, die entweder dem sofortigen weiteren Aufbau und Wachstum des Organismus dienen oder gespeichert werden (z. B. Glukose/Speicherfette). Wesentlich für den Stoffwechsel sind Enzyme, die chemische Reaktionen katalysieren.

Katabole wie anabole Stoffwechselzustände ( Kasten) sind mit einer spezifischen Hormonkonstellation verbunden.
Für den kontrollierten rhythmischen Wechsel zwischen katabolem und anabolem Stoffwechsel müssen nach Ansicht von Schole immer mehrere Hormone gleichzeitig und in der richtigen Zusammensetzung im Zytoplasma und Zellkern vorliegen. Das zeigt deutlich, dass die jeweilige dominierende Stoffwechselausrichtung auch immer an eine spezielle Hormonkonstellation gekoppelt ist, die, entsprechend ihrer Aufgabe von Aufbau/Aktivierung und Abbau/Deaktivierung, einen katabolen bzw. anabolen Stoffwechselzustand beschreibt.

Gesteuert über die wechselnden Quantitäten der anabolen bzw. katabolen Peptide und Hormone ereignet sich ein natürlicher rhythmischer Wechsel kataboler und anaboler Zustände: Rhythmischer „Stoff-Wechsel“ durch vernetztes hormonelles Wirken!

\section{Stoffwechselzustände}

\section{Katabolismus}

Als Katabolismus bezeichnet man die Reaktionen des Stoffwechsels, die dem Abbau chemischer Verbindungen dienen. Im Zuge der katabolen Reaktionen werden im Idealfall die über die Nahrung aufgenommenen Kohlenhydrate und Fette abgebaut (verbrannt), bei leeren Glykogenspeichern zusätzlich auch Eiweißgewebe (Glukoneogenese). Im katabolen Zustand sind im Körper Kortison und T3/4 sowie katabole Peptide erhöht. Sie verursachen eine Auflösung und dadurch den Verbrauch von Materie. Dies dient der Bereitstellung von Energie für den anabolen Prozess.

\section{Anabolismus}

Als Anabolismus bezeichnet man die Reaktionen des Stoffwechsels, die dem Aufbau chemischer Verbindungen, körpereigener Substanzen und Strukturen dienen. Im anabolen Zustand sind im Körper somatotrope Wachstumsfaktoren (STH) und anabole Peptide erhöht. Die Aufbauprozesse erfordern die Bereitstellung von Materie und Energie. 


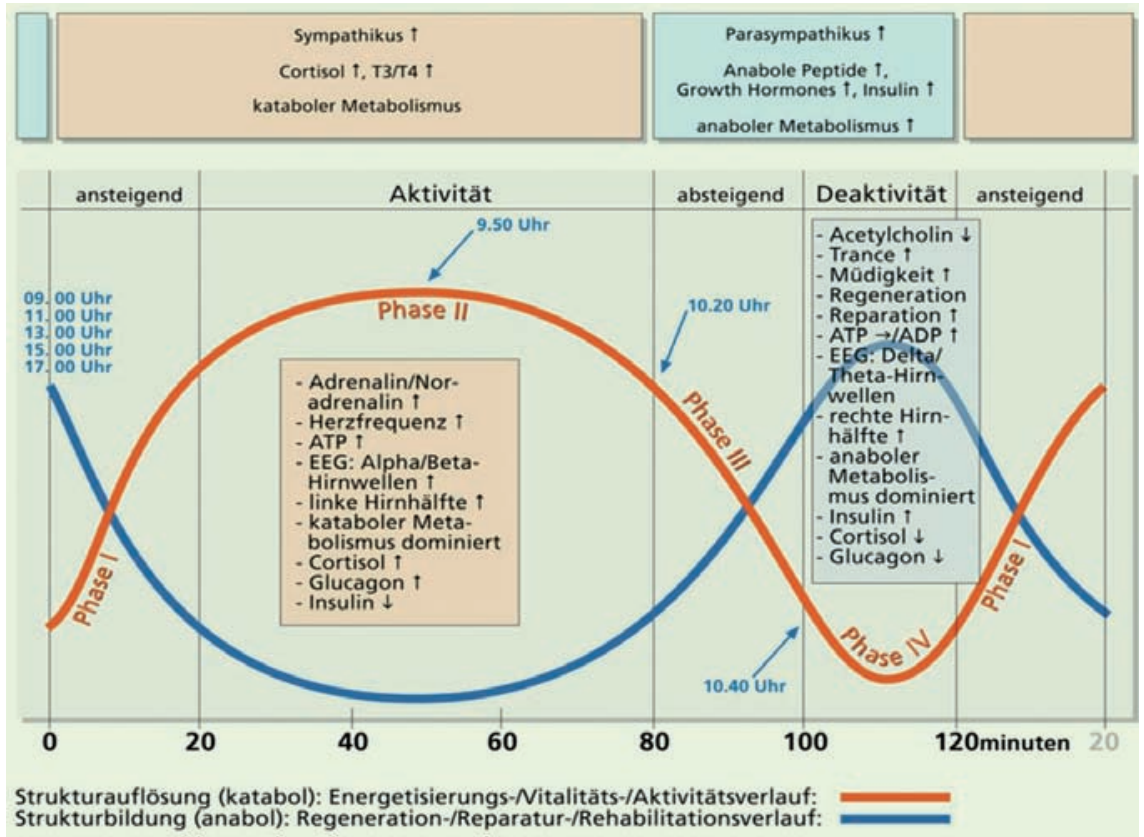

Abb. 2 Die Phasen des BRAC in der Übersicht. Foto: (c) A. Heinen

Kataboler und anaboler Zellstoffwechsel bestimmen zudem die Oxidations- und Reduktionsprozesse, also die Abgabe und Aufnahme der Elektronen und Protonen. Das Fehlen oder das Hinzufügen nur eines dieser Ladungsteilchen hat bereits Einfluss auf den $\mathrm{pH}-$ Wert und damit auf den chemischen Ablauf enzymatischer Prozesse. Die anatomische Struktur der Matrix ist Spannungsfeld und Leitsystem für den Ladungsaustausch und steuert die regulativen Prozesse auf dieser Ebene wesentlich schneller als z. B. die enzymatischen Prozesse.

Neubildung und Wiederauflösung von Strukturen, Aufrechterhaltung der verlustfreien Spannung, die Informationsvermittlung und die Kontrolle der Ladungsträger durch Zellstoffwechsel sowie die SäureBasen-Regulation: Dies alles geschieht in vernetzten rhythmisch-abhängigen biochemischen Prozessen der Matrix. Störungen im Rhythmusablauf oder gar der Verlust dieses Rhythmusspiels durch lang andauernde oder intensiv erhöhte Stresseinwirkungen verhindern die physiologische Regulation (Adaptation). Krankheiten können entstehen.

\section{Der Biologische-Ruhe-Aktivitäts- Cyklus}

Der nachfolgend beschriebene Cyklus, der sich im Abstand von 2 Stunden periodisch wiederholt und organismusspezifische biologische Rhythmen des metabolischen Ge- schehens darstellt, wird in der Medizin auch als Biologischer-Ruhe-Aktivitäts-Cyklus (BRAC) beschrieben ( Abb. 2). Der BRAC lässt sich in 4 Phasen aufteilen und ist als der spezifische Ruhe-Rhythmus des menschlichen Organismus zu betrachten. Er steuert koordiniert grundlegende Funktionen der Matrix und somit des Organismus. Die folgende Beschreibung nach A. Heinen dient dem weiteren Verständnis der vernetzten Regulationsabläufe der Matrix.

\section{BRAC-Phase 1}

Diese Phase ist die Anstiegsphase (Dauer ca. $20 \mathrm{~min}$ ). Sie zeigt einen dominant katabolen Stoffwechsel mit folgender Hormonkonstellation:

- Konzentration der Hormone Adrenalin/Noradrenalin steigt zunehmend (Sympathikus dominiert)

- Konzentration von Glukagon/ Glukokortikoiden/Aldosteron/und ADH steigt zunehmend

- Insulin wird zunehmend supprimiert

- Zahl der T-Helfer-Zellen wird zunehmend supprimiert, die Zahl der T-Suppressor-Zellen steigt

- es entstehen infolge der dominierenden Oxidationsprozesse auf natürliche Weise Ozon-Moleküle und Radikale $\left(\mathrm{O}^{-}\right) \rightarrow$ günstige Abwehrhilfe! (zerstören Bakterien und Viren) - verstärkte Säurebildung $\left(\mathrm{H}^{+}\right.$-Ionen, pH-Wert $\uparrow$ )

\section{BRAC-Phase 2}

Diese Phase ist die Aktivitätsphase (Dauer ca. $60 \mathrm{~min}$ ). Die dominant katabolen Stoffwechselvorgänge werden zunehmend durch anabole Stoffwechselvorgänge ergänzt, um die Vitalfunktionen stabil zu halten. Für die Hormonkonstellation ergibt sich folgender Zustand:

- Konzentration antiinsulinärer Hormone (Glukagon, Kortison) steigt

n relativer Insulinmangel/Insulinresistenz

- Konzentration der Wachstumsfaktoren ist in Relation zur Konzentration der katabolen Peptide gesteigert

- Konzentration von ADH/Aldosteron nimmt ab, ist aber weiterhin erhöht

- Sympathikus dominiert weiterhin gegenüber Parasympathikus

- Zahl der T-Helfer-Zellen steigt, die Zahl der T-Suppressor-Zellen fällt zunehmend

- Sauerstoffverbrauch steigt

- erhöhte ATP-Produktion

- Säure-Basen-Verhältnis: von der Säure aus zur Base ansteigend annähernd im Verhältnis 1:1

- Oxidation-Reduktion: von der Oxidation aus zur Reduktion ansteigend annähernd im Verhältnis 1:1

- es entstehen überwiegend SingulettSauerstoff-Moleküle

\section{BRAC-Phase 3}

Diese Phase ist die Abstiegs-Phase (Dauer ca. $20 \mathrm{~min}$ ). In dieser Phase überwiegt der Einfluss der anabolen Peptide und der Wachstumsfaktoren, sodass ein fast ausschließlich anaboler Zustand erreicht wird. Es ergibt sich nun folgende Hormonkonstellation:

- Insulin wird zum dominierenden Hormon (Hyperinsulinämie)

n antiinsulinäre Hormone (Kortison/ Glukagon) sind im Referenzbereich

- Konzentration der Wachstumsfaktoren und anaboler Peptide steigt an, jedoch durch die Hyperinsulinämie nur reduziert; anfangs zuviel, später zu wenig Insulin

- Konzentration von ADH/Aldosteron reduziert

- Zahl der T-Helferzellen ist deutlich erhöht gegenüber der T-SuppressorZellen und mobilisierten Antikörper

- Parasympathikus beginnt gegenüber dem Sympathikus zu dominieren 
- fast ausschließlich reduktive Stoffwechselprozesse

- fast ausschließlich Basenbildung (e-)

- hoher Sauerstoff- und Vitamin-BVerbrauch, da ausgeprägt laufender Zitratzyklus, um genügend ATP für den Strukturaufbrauch zu liefern; auch hoher Verbrauch an $\mathrm{Ca}^{2+}$ und $\mathrm{Mg}^{2+}$

\section{BRAC-Phase 4}

Diese Phase ist die Deaktivitäts-Phase (Dauer ca. 20 min). Die katabolen Vorgänge in dieser Rehabilitationsphase finden hauptsächlich als Energie-(ATP)-bildende Prozesse statt, zum Wiederaufbau und Auffüllen der während der Aktivität geleerten Glykogen- und Fettspeicher. Ein Zuviel an ATP wird in ADP umgewandelt. Nach den dominant oxidativ abgelaufenen Prozessen finden jetzt dominant reduktive Prozesse statt.

- Konzentration von Adrenalin/ Noradrenalin ist deutlich gegenüber Acethylcholin erniedrigt, der Parasympathikus dominiert

- Konzentration von Glukagon/ Glukokortikoiden/Aldosteron/ und ADH sinkt in den Normwertbereich

- Insulinspiegel leicht erhöht bis normal

n die T-Helfer-Zellen werden verstärkt durch T-Suppressor-Zellen neutralisiert

- verminderter Sauerstoffverbrauch
- Säure-Basen-Verhältnis: von der Base aus zur Säure ansteigend annähernd im Verhältnis 1:1

- Oxidation-Reduktion: von der Reduktion aus zur Oxidation ansteigend annähernd im Verhältnis 1:1

- es entstehen überwiegend ionisierte Sauerstoff-Moleküle

\section{Fazit für die Praxis}

Betrachtet man dieses Ruhe-RhythmusModell, kann der Stellenwert einer therapeutischen Beeinflussung des Grundregulationssystems nicht hoch genug eingeschätzt werden. Nach Köhler sollte sich jeder Therapeut vor einer Behandlung daher folgende Fragen stellen:

- Gibt es noch einen funktionierenden natürlichen Rhythmus (Dauerstress)?

- Welche Regulationsmechanismen haben versagt (anabole oder katabole Entgleisung/Rhythmusverlust)?

- Wer oder was hat die Einheit gestört (Integration oder Separation)?

- Woran mangelt es (Hormone, Enzyme, Nährstoffe, Elektronen/Protonen)?

Dieser Artikel ist online zu finden unter: http://dx.doi.org//10.1055/s-0030-1270386

\section{Weiterführende Literatur}

[1] Heinen A. Experimentelle Ergebnisse und Gedanken zu einem neuen ultraschnellen
Informations- und Regelsystem-Modell für den menschlichen Körper. Kongress: Neue Wege in der Medizin, Synergien zwischen Schulmedizin und ganzheitlicher Medizin Impulse zu neuem Denken. Glarus (Schweiz) im September 2001: Kongressband

[2] Heine $\mathbf{H}$. Lehrbuch der biologischen Medizin. Stuttgart: Hippokrates; 2007

[3] Köhler B. Biophysikaliche InformationsTherapie. Niebüll: Videel; 2003

\section{(a) Internet}

www.stressexperte.de

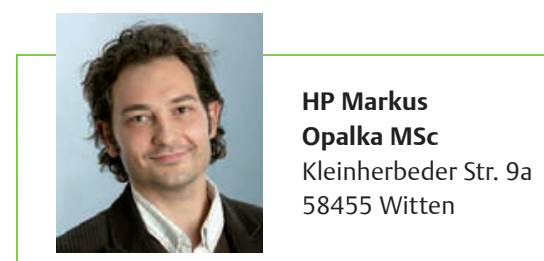

Markus Opalka ist niedergelassen in eigener Naturheilpraxis in Witten (NRW). Als Heilpraktiker arbeitet er mit dem Schwerpunkt Osteopathie, insbesondere für Säuglinge/Kinder, und mit Methoden der Regulationsmedizin. Darüber hinaus ist er als Dozent im Bereich Integrative Medizin im In- und Ausland tätig. Seit 2006 ist er Herausgeber der DHZ und arbeitet als Gesundheitswissenschaftler in weiteren Forschungsprojekten.

E-Mail: post@markusopalka.de 